\title{
DER FÜR DIE PRIESTERWAHLEN VORGESCHRIEBENE ZEITPUNKT
}

\author{
F. X. Ryan
}

Es ist 155 Jahre her, daß die Forschung verfügt hat, die Wahl der Priester habe man unmittelbar vor der Wahl der Prätoren durchzuführen. Hervorgehen soll dies aus einem Briefe, in welchem Cicero, der seinem Sohne die Aufnahme in das Pontifikat verschaffen wollte, sich dahin gehend äußert, daß alles fast recht zügig vonstatten gegangen wäre, wenn C. Pansa (Cos. 43) überlebt hätte: omnino Pansa vivo celeriora omnia putabamus. statim enim collegam sibi subrogavisset; deinde ante praetoria sacerdotum comitia fuissent. nunc per auspicia longam moram video (Cic. ad M. Brut. 1.5.4). "Die Comitien zur Priesterwahl," so Mercklin, "waren durch den Tod beider Consuln verhindert. Wir ersehen daraus, daß diese sie leiteten, und erfahren zugleich, daß sie vor der Prätorwahl stattfanden. “"

Freilich zeigt der Brief, daß die sacerdotum comitia früher stattfanden als die praetoria. Daß die sacerdotum comitia in der Tat später stattfanden als die consularia, zeigt der Brief jedoch nicht. Der Brief kann gar nicht beweisen, was er beweisen soll, denn bei den Konsularkomitien, auf welche Cicero anspielt, handelt es sich nicht um die Wahl zweier Konsuln, sondern um die Nachwahl eines einzigen Konsuls. Es nimmt nun nicht wunder, daß Cicero die Wahl der Konsuln für das Jahr 42 mit keinem Wort erwähnt: Wir wissen nämlich, daß der Diktator Caesar nicht nur sämtliche Magistrate für das Jahr 43, sondern auch für das Jahr darauf die Konsuln $^{2}$ und die Volkstribune ${ }^{3}$ wählen ließ (Cic. Att. 14.6.2, Dio 43.51.6). Man könnte zwar als aufschlußreich hinstellen, daß die Priesterwahlen jedenfalls auf die Wahl zum Suffektkonsul folgten, aber man müßte für möglich, ja sogar wahrscheinlich halten, daß eine Wahl für das aktuelle Jahr denen für das kommende Jahr stets voraufging. Es ließe sich denken, daß die Wahl zum Suffektprätor ebenfalls den Priesterwahlen voraufgegangen wäre, wie diese ihrerseits der Wahl zum Prätor voraufgingen. Wenn man nun von dem Datum des Briefes, dem 5. Mai, Kenntnis nimmt, wird man ohnehin zum Schluß gezwungen, daß Cicero hier zwei ganz verschiedene Wahltermine in Betracht zieht, die sofortige (statim) Wahl eines Suffektkonsuls, wohl noch im Mai, und die Jahrwahlen zur gewohnten Zeit, Ende Juli bzw. Anfang August; der Zeitraum, den deinde signalisiert, beträgt damit wenigstens anderthalb Monate (Anfang Juni - Mitte Juli).

\footnotetext{
${ }^{1}$ Ludwig Mercklin, Die Cooptation der Römer. Eine sacralrechtliche Abhandlung, Mitau und Leipzig 1848, 147-48. Vgl. T. Mommsen, Römisches Staatsrecht, Leipzig 1887, $2^{3} .32$ : ,zwischen denen der Consuln und der Prätoren“; G. Wissowa, Religion und Kultus der Römer, 2. Aufl., München 1912, 488 A. 6: „zwischen den Consular- und Praetorencomitien“; L. R. Taylor, Caesar's Colleagues in the Pontifical College, AJP 63, 1942, 388: „once a year, apparently between the consular and the praetorian comitia”; T. Robt. S. Broughton, Candidates Defeated in Roman Elections: Some Ancient Roman "Also-Rans," Philadelphia 1991, 50: "in the interval between those for consuls and those of praetors." Auch ein so ausgewiesener Kenner der römischen Verfassung und Priestertümer, wie es Herr Linderski ist, sagte sich von diesem Glauben nicht los; s. J. Linderski, The Aedileship of Favonius, Curio the Younger and Cicero's Election to the Augurate, HSCP 76, 1972, 192 (= Roman Questions, Stuttgart 1995, 242): ,at a stated time, between the consular and the praetorian elections.“

${ }^{2}$ Darum ist L. Munatius Plancus (Cos. 42), der am 17. März 44 im Senate vor den gewesenen Konsuln gesprochen zu haben scheint, ,the only consul-designate seen speaking not in but before the year preceding the year for which he had been designated“" (s. F. X. Ryan, Rank and Participation in the Republican Senate, Stuttgart 1998, 248).

${ }^{3}$ Darum tauchen designierte Volkstribune schon in der ersten Hälfte d. J. 43 auf; s. unsren Anhang, Three Caesarian Tribunes-Designate, in: Athenaeum 85, 1997, 263.
} 
In der uns interessierenden Frage ist ein Brief des Caelius aus dem Jahre 51 nicht eindeutiger als der Brief Ciceros: Er erwähnt zwar die Konsulwahlen vor den Priesterwahlen, wer aber diese Aussagen in ihrem Zusammenhang sieht — aufgezeichnet werden der Freispruch für einen Mann, dann die Verurteilung desselben; ein einziger Sieger bei den Konsulwahlen; ein Verlierer bei den Konsulwahlen, der danach angeklagt wurde; der Siegende und der Besiegte bei der Wahl zum Quindecimvir (Cael. Fam. 8.4.1) - , der wird schwerlich behaupten, die Reihenfolge der Berichterstattung müsse der der Ereignisse entsprechen. Als Caelius nun den Brief am 1. August schrieb, stand die Wahl der Prätoren noch bevor (Fam. 8.4.3: praetoriis morae quaedam inciderunt). Mit Sicherheit werden wir also diesem Brief nur das entnehmen können, was wir schon wußten, daß nämlich die Prätorenkomitien später stattfanden als die Priesterkomitien. Keine andere Quelle hilft uns weiter. ${ }^{4}$

Das, was in Ermangelung der Konsulwahlen vor den Prätorenwahlen erfolgte, könnte man sonst vor den Konsulwahlen erledigt haben. Wir können froh sein, daß Caesar nicht auch die Prätoren des Jahres 42 wählen ließ: In diesem Fall hätte Cicero ,ante comitia aed. cur.“ schreiben müssen, und wir müßten drei Möglichkeiten abwägen. Es hätte also schlimmer kommen können. Weder die eine noch andere Ansicht kann nachgewiesen werden, doch es dürfte schwierig sein, die Mercklins auch nur wahrscheinlich zu machen. Daß man die Priesterwahlen vor den eigentlichen Magistratswahlen abzuhalten hatte, anstatt diese durch jene zu unterbrechen, liegt, wie uns scheint, am nächsten. Dafür spricht auch, daß man sich so bemüht hätte, die vakant gewordenen Posten vor den vakant werdenden zu besetzen.

Die eine Regel Mercklins - der Konsul habe die Priesterwahlen geleitet - verbietet uns indes, die andere ganz abzuschaffen. Man wird nämlich weiterhin davon ausgehen können, daß es dem Interrex oblag, die Wahl möglichst der beiden Konsuln zu vollziehen, während es einem der neuen Konsuln zufiel, die Wahlen für das laufende Jahr weiterzuführen. ${ }^{5}$ Mithin gingen doch beim Interregnum die Konsulwahlen den Priesterwahlen, die Priesterwahlen ihrerseits den Prätorenwahlen vorauf. ${ }^{6}$ Die Reihenfolge, die man bisher für den Normalfall hielt, war also zumindest eine seltene Ausnahme. ${ }^{7}$ Bekanntlich war die Versammlung der siebzehn ausgelosten Tribus erst ab dem Jahr 104, nicht mehr aber in der Zeit zwischen 82 und 63 für die Priesterbestellung zuständig, und zu Interregna kam es zumeist nicht: Zur Zeit der Republik wurden daher die Priesterwahlen höchstens dreimal (55, 53 und 52 v. Chr.) zwischen den Konsul- und den Prätorenwahlen nachgeholt. Mercklins Reihenfolge ist, wohlgemerkt, unter der Wahlleitung ein und desselben Mannes kein einziges Mal Wirklichkeit geworden. ${ }^{8}$

\section{Dr. Francis Xavier Ryan \\ Technische Universität Dresden, Institut für Geschichte, Lehstuhl für Alte Geschichte E-mail:fxryan@gmx.de}

\footnotetext{
${ }^{4}$ In seinem Bericht über d. J. 49 erwähnt Dio (41.36.2-3) die Ergänzung der Priesterschaften erst nach der Besetzung der Jahresämter; eine Bemerkung Senecas (de Benef. 7.28.2) besagt nur, daß ein und derselbe Mann erheblich später für das Konsulat oder eine Priesterstelle kandidierte als die Quästur.

${ }^{5}$ Vgl. etwa W. Kunkel, Staatsordnung und Staatspraxis der römischen Republik. Die Magistratur, München 1995, 86 A. 113: „Stets offen blieben bei der Interregnalwahl die Magistraturen von der Prätur abwärts.“

${ }^{6}$ Prosopographisch ist dies nicht ohne Belang. Die Annahme Linderskis (Cicero's Election, S. 193, 199), Cicero habe das Augurat frühestens im Juli 53 bzw. im März 52 erlangt, d.h., daß die Wahl zum Konsul jeweils den oberen Terminus für die Wahl zum Augur darstelle, wird nicht hinfällig, da in den fraglichen Jahren das Interregnum eingetreten war.

${ }^{7}$ Eine Ausnahme wurde aber i. J. 43, als es streng genommen keine Interreges gab, nicht gemacht, da die Priesterwahlen zunächst in alterum annum vertagt wurden (Cic. ad M. Brut. 1.14.1); vgl. MRR 2.356-57.

${ }^{8}$ Für die Vergabe eines Forschungsstipendiums sind wir der Alexander von Humboldt-Stiftung zu besonderem Danke verpflichtet.
} 